\title{
PHASE CHANGES IN THE TOPOLOGICAL INDICES OF SCALE-FREE TREES
}

\author{
QUNQIANG FENG *** AND \\ ZHISHUI HU, ${ }^{* * *}$ University of Science and Technology of China
}

\begin{abstract}
A scale-free tree with the parameter $\beta$ is very close to a star if $\beta$ is just a bit larger than -1 , whereas it is close to a random recursive tree if $\beta$ is very large. Through the Zagreb index, we consider the whole scene of the evolution of the scale-free trees model as $\beta$ goes from -1 to $+\infty$. The critical values of $\beta$ are shown to be the first several nonnegative integer numbers. We get the first two moments and the asymptotic behaviors of this index of a scale-free tree for all $\beta$. The generalized plane-oriented recursive trees model is also mentioned in passing, as well as the Gordon-Scantlebury and the Platt indices, which are closely related to the Zagreb index.
\end{abstract}

Keywords: Random network; small world; Zagreb index; scale-free tree

2010 Mathematics Subject Classification: Primary 05C05; 60C05

Secondary 60F05

\section{Introduction}

Recently, random networks have been a very popular research area among physicists and mathematicians. A lot of effort has been put into the investigation of such systems, in order to recognize their structures and to analyze emerging complex properties. It was observed that most real-world networks share three prominent structural features: the small-world property, high clustering, and scale-free degree distribution (see, for example, Dorogovtsev and Mendes (2003)).

The scale-free trees model is one of the fundamental dynamics networks, which was introduced in Barabási and Albert (1999). In the beginning, we only have a single node labeled 0. Progressively, nodes are added in steps: at the $n$th step a node in the existing tree is chosen as the parent of the new node (labeled $n$ ). The probability that a given old node is chosen is proportional to its degree. This principle of adding nodes is called preferential attachment. Móri (2002) generalized this model by replacing the node degree with a fixed linear function (weight) of itself. Formally, the probability that a given old node is chosen is $(k+\beta) / S_{n-1}$, where $\beta>-1$ is a given real number, $k$ is the degree of the node, and

$$
S_{n}=2 n+(n+1) \beta=(2+\beta) n+\beta
$$

is the sum of all the weights after the $n$th step. Clearly, the special case $\beta=0$ is the BarabásiAlbert random tree.

Received 23 February 2012.

* Postal address: Department of Statistics and Finance, School of Management, University of Science and Technology of China, Hefei, Anhui 230026, China.

** Email address: fengqq@ustc.edu.cn

*** Email address: huzs@ustc.edu.cn 
The degree distribution of a scale-free tree is a power law (see Móri (2002)), like most of the real-world networks (see, for example, Bollobás et al. (2001)). Móri (2005) also studied the convergence of the joint degree distribution as well as the limiting distribution of the maximal degree. The spanning trees of various real-world networks are also scale-free trees (see Kim $e t$ al. (2004)). For recent works on the scale-free trees model and more background, we refer the reader to Katona (2005), (2006), and Dondajewski and Szymański (2009).

The Zagreb index, one of the well-known topological indices in computational chemistry, was introduced by the chemists Gutman and Trinajstić (1972). This index of a (chemical) graph is defined as the sum of the squares of the degrees of all vertices in the graph. In chemistry, graphs are generated from molecules by replacing atoms with vertices and bonds with edges, or represent only bare molecular skeletons, that is, molecular skeletons without hydrogen atoms. For a survey of the application of the Zagreb index in computational chemistry, we refer the reader to Nikolić et al. (2003) and the references therein. The Zagreb index also attracts attention in graph theory (see, for example, Andova et al. (2011), Nikiforov (2007), and Peled et al. (1999)).

The small-world property in random networks is usually described as the average path length (APL) between nodes is small. However, both the APLs of a scale-free tree and a random recursive tree increase approximately logarithmically with the tree size $n$ (see Durrett (2007) or Bollobás and Riordan (2004) for the scale-free trees and Neininger (2002) for random recursive trees), while the degree distribution of a random recursive tree is not a power law but the geometric distribution (see, for example, Janson (2005)). Since the nodes with large degrees will shorten the APL, there exists some inner connection between the small-world property and the degree distribution. We propose the Zagreb index as a bridge between them. For any tree, the total sum of the degrees of its nodes is determinately equal to twice the nodes number minus two. But if we consider the sum of squares of degrees in a random tree, it is now a random variable. The nodes with large degrees, if they exist, will contribute mainly to this quantity, whereas this index will be small if the nodes have almost common degrees. That is, the effect of the nodes with large degrees is magnified by the Zagreb index. For a tree $T$ with size $n, \mathrm{Li}$ et al. (2003) studied the extreme values of the Zagreb index of $T$ : it reaches the minimum value $4 n-6$ if and only if $T$ is a path (with the longest APL) on $n$ nodes, and reaches the maximum value $n(n-1)$ if and only if $T$ is a star (with the shortest APL) on $n$ nodes.

Feng and $\mathrm{Hu}$ (2011) showed that a random recursive tree is much 'closer' to a path than to a star by considering its Zagreb index: the Zagreb index of a random recursive tree is approximately $6 n$. One can imagine the following fact. A scale-free tree with $\beta$ close to -1 , say $\beta=-0.99$, is very close to a star, whereas it is close to a random recursive tree if $\beta$ is very large. Our aim is to study the phase changes in the Zagreb indices of scale-free trees. We will show that the critical values of $\beta$ are the first several nonnegative integer numbers.

For our work, it is easier to deal with the modified Zagreb index that is defined in exactly the same way as the standard index, except that all the degrees of the nodes are changed to their weights. Mathematically, for a scale-free tree after the $n$th step, we can define the general Zagreb and modified Zagreb indices as

$$
Z_{n}^{(m)}=\sum_{k=0}^{n} D_{n, k}^{m}, \quad X_{n}^{(m)}=\sum_{k=0}^{n}\left(D_{n, k}+\beta\right)^{m}, \quad m=2,3, \ldots,
$$

where $D_{n, k}$ denotes the degree of node $k$. For the standard cases $m=2$, we will suppress the superscripts. 
Throughout, we will use the following notation. Let us denote by $H_{n}$ the $n$th harmonic number (i.e. $H_{0}=0$ and $H_{m}=\sum_{i=1}^{m} 1 / i$ for $m \geq 1$ ), the integer part of a real number $x$ by $\lfloor x\rfloor$, and, for nonnegative integers $n$ and $k$,

$$
c[n, k]:=\frac{\Gamma(n+\beta /(2+\beta))}{\Gamma(n+(k+\beta) /(2+\beta))} .
$$

For any fixed $k>0$, it is easy to see that $c[n, k]$ is strictly decreasing,

$$
\frac{c[n+1, k]}{c[n, k]}=\frac{S_{n}}{S_{n}+k}
$$

and, as $n \rightarrow \infty$,

$$
c[n, k]=n^{-k /(2+\beta)}\left(1+O\left(\frac{1}{n}\right)\right) .
$$

Our main results are the following two theorems.

Theorem 1. Let $Z_{n}$ be the Zagreb index of a scale-free tree after the nth step. As $n \rightarrow \infty$, the following assertions hold.

(i) If $\beta>2$ then

$$
\frac{Z_{n}-(6+4 / \beta) n}{\sqrt{n}} \stackrel{\mathrm{D}}{\rightarrow} N\left(0, \frac{8(1+\beta)^{2}(2+\beta)^{2}}{(\beta-2)(\beta-1) \beta^{2}}\right)
$$

(ii) If $\beta=2$ then

$$
\frac{Z_{n}-8 n}{\sqrt{72 n \log n}} \stackrel{\mathrm{D}}{\rightarrow} N(0,1) .
$$

(iii) If $-1<\beta<2$ then there exists a random variable $Z$ with $\mathbb{E}[Z]^{2}<\infty$ such that

$$
c[n, 2]\left(Z_{n}-\mathbb{E}\left[Z_{n}\right]\right) \rightarrow Z \text { almost surely (a.s.) and in } L^{2},
$$

where $\mathbb{E}\left[Z_{n}\right]$ is given in (6) below.

As the calculations of the moments of $Z_{n}$ in the next section reveal, Theorem 1 shows that the normalized version $\left(Z_{n}-\mathbb{E}\left[Z_{n}\right]\right) / \sqrt{\operatorname{var}\left[Z_{n}\right]}$ tends to a limit, which is normal for $\beta \geq 2$ and a nondegenerate limit for $-1<\beta<2$. For many real-world networks, the parameter $\beta$ is small or even negative (see, for example, Albert and Barabási (2002)). Then it is necessary to consider the further limiting behavior for the case $-1<\beta<2$.

Theorem 2. As $n \rightarrow \infty$, the following assertions hold.

(i) If $1<\beta<2$ then

$$
n^{(2-\beta) /(2 \beta+4)}\left(c[n, 2]\left(Z_{n}-\mathbb{E}\left[Z_{n}\right]\right)-Z\right) \stackrel{\mathrm{D}}{\rightarrow} N\left(0, \frac{8(1+\beta)^{2}(2+\beta)^{2}}{(2-\beta)(\beta-1) \beta^{2}}\right) .
$$

(ii) If $\beta=1$ then

$$
\frac{n^{1 / 6}}{\sqrt{96 \log n}}\left(c[n, 2]\left(Z_{n}-\mathbb{E}\left[Z_{n}\right]\right)-Z\right) \stackrel{\mathrm{D}}{\rightarrow} N(0,1) .
$$


(iii) If $-1<\beta<1$ then

$$
n^{1 /(2 \beta+4)}\left(c[n, 2]\left(Z_{n}-\mathbb{E}\left[Z_{n}\right]\right)-Z\right) \stackrel{\mathrm{D}}{\rightarrow} N\left(0,4\left(\tilde{Z}+C_{2}\right)\right),
$$

where the constant $C_{2}$ is defined in (8) below,

$$
\tilde{Z}=\lim _{n \rightarrow \infty} c[n, 3]\left(Z_{n}^{(3)}-\mathbb{E}\left[Z_{n}^{(3)}\right]\right) \text { a.s., }
$$

and the normal mixture $N\left(0,4\left(\tilde{Z}+C_{2}\right)\right)$ is defined as the distribution of the product $2 \sqrt{\tilde{Z}+C_{2}} \mathcal{N}$ with a standard normal random variable $\mathcal{N}$, independently of $\tilde{Z}$.

The sequel is organized as follows. The first two moments of the Zagreb index of a scale-free tree after the $n$th step are given in Section 2. We introduce several lemmas and give the proofs of the main theorems in Section 3. In the last section, a related random trees model and two topological indices related to the Zagreb index are mentioned in passing.

\section{The moments}

Let the random variables $Z_{n}$ and $X_{n}$ respectively be the standard and modified Zagreb indices of a scale-free tree after the $n$th step. Let $D_{n, k}$ denote the degree of node $k$ (i.e. $W_{n, k}:=D_{n, k}+\beta$ is the weight of node $k$ ), and let $V_{n}$ denote the label of the chosen node (the parent of the new node) in the scale-free tree at the $n$th step. Note that the total sum of the degrees of the nodes in a tree with size $n$ is $2(n-1)$. Then

$$
\begin{aligned}
X_{n} & =\sum_{k=0}^{n} W_{n, k}^{2} \\
& =\sum_{k=0}^{n}\left(D_{n, k}+\beta\right)^{2} \\
& =\sum_{k=0}^{n} D_{n, k}^{2}+2 \beta \sum_{k=0}^{n} D_{n, k}+(n+1) \beta^{2} \\
& =Z_{n}+\left(4 \beta+\beta^{2}\right) n+\beta^{2} .
\end{aligned}
$$

We also define $\mathcal{F}_{n}$ to be the $\sigma$-field generated by the first $n$ steps of the scale-free trees.

Considering the insertion of node $n$ at the $n$th step, we obtain

$$
\begin{aligned}
X_{n} & =X_{n-1}+\left(W_{n-1, V_{n}}+1\right)^{2}-W_{n-1, V_{n}}^{2}+(1+\beta)^{2} \\
& =X_{n-1}+2 W_{n-1, V_{n}}+2+2 \beta+\beta^{2} .
\end{aligned}
$$

Then

$$
\begin{aligned}
\mathbb{E}\left[X_{n} \mid \mathcal{F}_{n-1}\right] & =X_{n-1}+2 \sum_{k=0}^{n-1} \mathbb{P}\left(V_{n}=k \mid \mathcal{F}_{n-1}\right) W_{n-1, k}+2+2 \beta+\beta^{2} \\
& =X_{n-1}+\frac{2}{S_{n-1}} \sum_{k=0}^{n-1} W_{n-1, k}^{2}+2+2 \beta+\beta^{2} \\
& =\left(1+\frac{2}{S_{n-1}}\right) X_{n-1}+2+2 \beta+\beta^{2} \\
& =\frac{c[n-1,2]}{c[n, 2]} X_{n-1}+2+2 \beta+\beta^{2} .
\end{aligned}
$$


Taking the expectation of both sides of (4), we have

$$
\mathbb{E}\left[X_{n}\right]=\frac{c[n-1,2]}{c[n, 2]} \mathbb{E}\left[X_{n-1}\right]+2+2 \beta+\beta^{2},
$$

which, with the initial value $\mathbb{E}\left[X_{0}\right]=\beta^{2}$, implies that

$$
\mathbb{E}\left[X_{n}\right]=\frac{c[0,2]}{c[n, 2]} \beta^{2}+\frac{2+2 \beta+\beta^{2}}{c[n, 2]} \sum_{i=1}^{n} c[i, 2], \quad n=1,2, \ldots
$$

By (1) we have

$$
\mathbb{E}\left[X_{n}\right]= \begin{cases}\left(\beta^{2}+4 \beta+6+\frac{4}{\beta}\right) n+O\left(n^{2 /(2+\beta)}\right), & \beta>0, \\ 2 n H_{n}=2 n \log n+O(n), & \beta=0, \\ C_{1} n^{2 /(2+\beta)}+O(n), & -1<\beta<0,\end{cases}
$$

where

$$
C_{1}:=c[0,2] \beta^{2}+\left(2+2 \beta+\beta^{2}\right) \sum_{n=1}^{\infty} c[n, 2]<\infty
$$

is a constant dependent only on $\beta$. By (2) we obtain the mean of $Z_{n}$.

Proposition 1. Let $Z_{n}$ be the Zagreb index of a scale-free tree after the nth step. Then

$$
\mathbb{E}\left[Z_{n}\right]= \begin{cases}\left(6+\frac{4}{\beta}\right) n+O\left(n^{2 /(2+\beta)}\right), & \beta>0, \\ 2 n H_{n}=2 n \log n+O(n), & \beta=0, \\ C_{1} n^{2 /(2+\beta)}+O(n), & -1<\beta<0 .\end{cases}
$$

An analogous technique to (3) gives

$$
\begin{aligned}
X_{n}^{(3)} & =X_{n-1}^{(3)}+\left(W_{n-1, V_{n}}+1\right)^{3}-W_{n-1, V_{n}}^{3}+(1+\beta)^{3} \\
& =X_{n-1}^{(3)}+3 W_{n-1, V_{n}}+3 W_{n-1, V_{n}}^{2}+1+(1+\beta)^{3} .
\end{aligned}
$$

Thus,

$$
\begin{aligned}
\mathbb{E}\left[X_{n}^{(3)} \mid \mathcal{F}_{n-1}\right] & =X_{n-1}^{(3)}+\frac{3}{S_{n-1}} \sum_{k=0}^{n-1}\left(W_{n-1, k}^{3}+W_{n-1, k}^{2}\right)+1+(1+\beta)^{3} \\
& =\left(1+\frac{3}{S_{n-1}}\right) X_{n-1}^{(3)}+\frac{3 X_{n-1}}{S_{n-1}}+1+(1+\beta)^{3} .
\end{aligned}
$$

Write

$$
b_{n}:=\frac{3 \mathbb{E}\left[X_{n-1}\right]}{S_{n-1}}+1+(1+\beta)^{3}, \quad n=1,2, \ldots
$$


Then

$$
\begin{aligned}
\mathbb{E}\left[X_{n}^{(3)}\right] & =\frac{c[n-1,3]}{c[n, 3]} \mathbb{E}\left[X_{n-1}^{(3)}\right]+b_{n} \\
& =\frac{c[0,3]}{c[n, 3]} \beta^{3}+\frac{1}{c[n, 3]} \sum_{i=1}^{n} c[i, 3] b_{i}, \quad n=1,2, \ldots
\end{aligned}
$$

By (5),

$$
b_{n}= \begin{cases}\beta^{3}+3 \beta^{2}+6 \beta+8+\frac{6}{\beta}+O\left(n^{-\beta /(2+\beta)}\right), & \beta>0, \\ 3 H_{n-1}+2=3 \log n+O(1), & \beta=0, \\ \frac{3 C_{1}(1+\beta)}{2+\beta} n^{-\beta /(2+\beta)}+O(1), & -1<\beta<0 .\end{cases}
$$

Hence,

$$
\mathbb{E}\left[X_{n}^{(3)}\right]= \begin{cases}\frac{(2+\beta)\left(\beta^{3}+3 \beta^{2}+6 \beta+8+6 / \beta\right)}{\beta-1} n+O\left(n^{3 /(2+\beta)}\right), & \beta>1, \\ 24 n \log n+O(n), & \beta=1, \\ C_{2} n^{3 /(2+\beta)}+O(n), & 0<\beta<1, \\ C_{2} n^{3 / 2}+O(n \log n), & \beta=0, \\ C_{2} n^{3 /(2+\beta)}+O\left(n^{2 /(2+\beta)}\right), & -1<\beta<0,\end{cases}
$$

where

$$
C_{2}:=c[0,3] \beta^{3}+\sum_{n=1}^{\infty} c[n, 3] b_{n}<\infty
$$

is a constant dependent only on $\beta$. Now, by (3),

$$
\mathbb{E}\left(X_{n}-X_{n-1}-\left(2+2 \beta+\beta^{2}\right)\right)^{2}=4 \mathbb{E}\left[W_{V_{n}, n-1}^{2}\right]=4 \sum_{k=1}^{n-1} \frac{\mathbb{E}\left[W_{n-1, k}^{3}\right]}{S_{n-1}}=\frac{4}{S_{n-1}} \mathbb{E}\left[X_{n-1}^{(3)}\right]
$$

It follows from (4) that the sequence $\left\{c[n, 2]\left(X_{n}-\mathbb{E}\left[X_{n}\right]\right), \mathcal{F}_{n}, n \geq 0\right\}$ is a martingale. Then

$$
\begin{aligned}
\mathbb{E}\left(X_{n}\right. & \left.-X_{n-1}-\left(2+2 \beta+\beta^{2}\right)\right)^{2} \\
& =\mathbb{E}\left(X_{n}-\mathbb{E}\left[X_{n}\right]-X_{n-1}+\mathbb{E}\left[X_{n-1}\right]\right)^{2}+\left(\mathbb{E}\left[X_{n}\right]-\mathbb{E}\left[X_{n-1}\right]-\left(2+2 \beta+\beta^{2}\right)\right)^{2} \\
& =\operatorname{var}\left[X_{n}\right]+\left(1-\frac{2 c[n-1,2]}{c[n, 2]}\right) \operatorname{var}\left[X_{n-1}\right]+4\left(\frac{\mathbb{E}\left[X_{n-1}\right]}{S_{n-1}}\right)^{2} .
\end{aligned}
$$

Hence,

$$
\operatorname{var}\left[X_{n}\right]=\left(\frac{2 c[n-1,2]}{c[n, 2]}-1\right) \operatorname{var}\left[X_{n-1}\right]+\hat{b}_{n}=\frac{c[n-1,4]}{c[n, 4]} \operatorname{var}\left[X_{n-1}\right]+\hat{b}_{n},
$$


where

$$
\begin{aligned}
\hat{b}_{n}: & =\frac{4}{S_{n-1}} \mathbb{E}\left[X_{n-1}^{(3)}\right]-4\left(\frac{\mathbb{E}\left[X_{n-1}\right]}{S_{n-1}}\right)^{2} \\
& = \begin{cases}\frac{8(1+\beta)^{2}(2+\beta)}{(\beta-1) \beta^{2}}+O\left(n^{(1-\beta) /(2+\beta)}\right), & \beta>1, \\
32 \log n+O(1), & \beta=1, \\
\frac{4 C_{2}}{2+\beta} n^{(1-\beta) /(2+\beta)}+O(1), & 0<\beta<1, \\
\frac{4 C_{2}}{2+\beta} n^{1 / 2}+O\left(\log ^{2} n\right), & \beta=0, \\
\frac{4 C_{2}}{2+\beta} n^{(1-\beta) /(2+\beta)}+O\left(n^{-2 \beta /(2+\beta)}\right), & -1<\beta<0 .\end{cases}
\end{aligned}
$$

For any $n \geq 0$, relation (2) implies that $\operatorname{var}\left[Z_{n}\right]$ is equal to $\operatorname{var}\left[X_{n}\right]$, which can be directly obtained from recurrence (9) with the initial values $\operatorname{var}\left[Z_{0}\right]=\operatorname{var}\left[Z_{1}\right]=0$.

Proposition 2. Let $Z_{n}$ be the Zagreb index of a scale-free tree after the nth step. Then

$$
\begin{aligned}
\operatorname{var}\left[Z_{n}\right] & =\operatorname{var}\left[X_{n}\right] \\
& =\frac{1}{c[n, 4]} \sum_{i=1}^{n} c[i, 4] \hat{b}_{i} \\
& = \begin{cases}\frac{8(1+\beta)^{2}(2+\beta)^{2}}{(\beta-2)(\beta-1) \beta^{2}} n+O\left(n^{4 /(2+\beta)}\right), & \beta>2, \\
72 n \log n+O(n), & \beta=2, \\
C_{3} n^{4 /(2+\beta)}+O(n), & 1<\beta<2, \\
C_{3} n^{4 / 3}+O(n \log n), & \beta=1, \\
C_{3} n^{4 /(2+\beta)}+O\left(n^{3 /(2+\beta)}\right), & 0<\beta<1, \\
C_{3} n^{2}+O\left(n^{3 / 2}\right), & \beta=0, \\
C_{3} n^{4 /(2+\beta)}+O\left(n^{(3-\beta) /(2+\beta)}\right), & -1<\beta<0,\end{cases}
\end{aligned}
$$

where $C_{3}=\sum_{n=1}^{\infty} c[n, 4] \hat{b}_{n}<\infty$ is a constant dependent only on $\beta$.

An immediate consequence of Proposition 1 and Proposition 2 is the following result.

Proposition 3. Let $Z_{n}$ be the Zagreb index of a scale-free tree after the nth step. If $\beta \geq 0$ then, as $n \rightarrow \infty$,

$$
\frac{Z_{n}}{\mathbb{E}\left[Z_{n}\right]} \stackrel{\mathbb{P}}{\rightarrow} 1 .
$$

Proof. For $\beta \geq 0$, it follows by Proposition 1 and Proposition 2 that $\operatorname{var}\left[Z_{n}\right]=o\left(\mathbb{E}^{2}\left[Z_{n}\right]\right)$, which implies the result via Chebyshev's inequality. 


\section{Proofs of the theorems}

In order to prove the main results, we need the following auxiliary lemmas.

Lemma 1. The following assertions hold.

(i) For any $\beta>-1$, the sequence $\left\{c[n, 2]\left(X_{n}-\mathbb{E}\left[X_{n}\right]\right), \mathcal{F}_{n}, n \geq 0\right\}$ is a martingale. Specifically, if $-1<\beta<2$, there exists a random variable $Z$ with $\mathbb{E}\left[Z^{2}\right]<\infty$ such that

$$
c[n, 2]\left(X_{n}-\mathbb{E}\left[X_{n}\right]\right)=c[n, 2]\left(Z_{n}-\mathbb{E}\left[Z_{n}\right]\right) \rightarrow Z \text { a.s. and in } L^{2} .
$$

(ii) Define

$$
T_{n}:=X_{n}^{(3)}+3 X_{n}-\mathbb{E}\left[X_{n}^{(3)}+3 X_{n}\right] .
$$

Then, for any $\beta>-1$, the sequence $\left\{c[n, 3] T_{n}, \mathcal{F}_{n}, n \geq 0\right\}$ is a martingale.

(iii) If $-1<\beta<2$, there exists a random variable $\tilde{Z}$ with $\mathbb{E}\left[\tilde{Z}^{2}\right]<\infty$ such that

$$
\lim _{n \rightarrow \infty} c[n, 3]\left(X_{n}^{(3)}-\mathbb{E}\left[X_{n}^{(3)}\right]\right)=\lim _{n \rightarrow \infty} c[n, 3]\left(Z_{n}^{(3)}-\mathbb{E}\left[Z_{n}^{(3)}\right]\right)=\tilde{Z} \quad \text { a.s. }
$$

Proof. It follows from (4) that the sequence $\left\{c[n, 2]\left(X_{n}-\mathbb{E}\left[X_{n}\right]\right), \mathcal{F}_{n}, n \geq 0\right\}$ is a martingale for any $\beta>-1$. For $-1<\beta<2$, Proposition 2 shows that

$$
\sup _{n \geq 0} \mathbb{E}\left(c[n, 2]\left(X_{n}-\mathbb{E}\left[X_{n}\right]\right)\right)^{2}<\infty .
$$

Then, by the martingale convergence theorem (see, for example, Theorem 2.5 and Corollary 2.2 of Hall and Heyde (1980)), assertion (i) holds by (2).

By (4) and (7), we have

$$
\begin{aligned}
\mathbb{E}\left[X_{n}^{(3)}+3 X_{n} \mid \mathcal{F}_{n-1}\right] & =\left(1+\frac{3}{S_{n-1}}\right)\left(X_{n-1}^{(3)}+3 X_{n-1}\right)+c_{0} \\
& =\frac{c[n-1,3]}{c[n, 3]}\left(X_{n-1}^{(3)}+3 X_{n-1}\right)+c_{0},
\end{aligned}
$$

where $c_{0}=1+(1+\beta)^{3}+3\left(2+2 \beta+\beta^{2}\right)$. Hence, assertion (ii) holds.

To prove assertion (iii), we first estimate the variance of $X_{n}^{(3)}+3 X_{n}$. Since $\left\{c[n, 3] T_{n}, \mathcal{F}_{n}\right.$, $n \geq 0\}$ is a martingale, an analogous technique as the calculation of $\operatorname{var}\left[X_{n}\right]$ gives, for $n \geq 1$,

$$
\operatorname{var}\left[T_{n}\right]=\operatorname{var}\left[X_{n}^{(3)}+3 X_{n}\right]=\frac{c[n-1,6]}{c[n, 6]} \operatorname{var}\left[X_{n-1}^{(3)}+3 X_{n-1}\right]+\tilde{b}_{n},
$$

where

$$
\tilde{b}_{n}:=\frac{9}{S_{n-1}} \mathbb{E}\left[X_{n-1}^{(5)}+6 X_{n-1}^{(4)}+9 X_{n-1}^{(3)}\right]-\frac{9}{S_{n-1}^{2}}\left(\mathbb{E}\left[X_{n-1}^{(3)}+3 X_{n-1}\right]\right)^{2}
$$

Let

$$
Z[n, k, 5]=c[n, 5]\left(\begin{array}{c}
W_{n, k}+4 \\
5
\end{array}\right) .
$$

By Theorem 2.1 of Móri (2005), the process $\left\{Z[n, k, 5], \mathcal{F}_{n}, n \geq k\right\}$ is a martingale and

$$
\mathbb{E}[Z[n, k, 5]]=\mathbb{E}[Z[k, k, 5]]=c[k, 5]\left(\begin{array}{c}
5+\beta \\
5
\end{array}\right) .
$$


This implies that

$$
\begin{aligned}
\tilde{b}_{n} & \leq \frac{9 \cdot 5 !}{S_{n-1}} \sum_{k=0}^{n-1} \mathbb{E}\left(\begin{array}{c}
W_{n, k}+4 \\
5
\end{array}\right) \\
& =O\left(\frac{n^{-1}}{c[n, 5]} \sum_{k=1}^{n} c[k, 5]\right) \\
& = \begin{cases}O(1), & \beta>3, \\
O(\log n), & \beta=3, \\
O\left(n^{(3-\beta) /(2+\beta)}\right), & \beta<3 .\end{cases}
\end{aligned}
$$

We thus have

$$
\operatorname{var}\left[X_{n}^{(3)}+3 X_{n}\right]=\frac{1}{c[n, 6]} \sum_{k=1}^{n} c[k, 6] \tilde{b}_{k}= \begin{cases}O(n), & \beta>4 \\ O(n \log n), & \beta=4, \\ O\left(n^{6 /(2+\beta)}\right), & \beta<4\end{cases}
$$

Then, for $-1<\beta<4$,

$$
\sup _{n \geq 0} \mathbb{E}\left[c[n, 3] T_{n}\right]^{2}<\infty .
$$

Therefore, also by the martingale convergence theorem, there exists a random variable $\tilde{Z}$ with $\mathbb{E}\left[\tilde{Z}^{2}\right]<\infty$ such that $c[n, 3] T_{n} \rightarrow \tilde{Z}$, a.s. and in $L^{2}$.

Note that

$$
c[n, 3]\left(X_{n}^{(3)}-\mathbb{E}\left[X_{n}^{(3)}\right]\right)=c[n, 3] T_{n}-3 c[n, 3]\left(X_{n}-\mathbb{E}\left[X_{n}\right]\right),
$$

and $c[n, 3] / c[n, 2] \rightarrow 0$ as $n \rightarrow \infty$. By assertion (i), we thus have, for $-1<\beta<2$,

$$
c[n, 3]\left(X_{n}^{(3)}-\mathbb{E}\left[X_{n}^{(3)}\right]\right) \rightarrow \tilde{Z} \quad \text { a.s. }
$$

An analogous calculation to (2) gives

$$
X_{n}^{(3)}=Z_{n}^{(3)}+3 \beta Z_{n}+6 n \beta^{2}+(n+1) \beta^{3},
$$

which implies that

$$
Z_{n}^{(3)}-\mathbb{E}\left[Z_{n}^{(3)}\right]=\left(X_{n}^{(3)}-\mathbb{E}\left[X_{n}^{(3)}\right]\right)-3 \beta\left(Z_{n}-\mathbb{E}\left[Z_{n}\right]\right) .
$$

Also, by assertion (i), for $-1<\beta<2$,

$$
\begin{aligned}
\lim _{n \rightarrow \infty} c[n, 3]\left(Z_{n}^{(3)}-\mathbb{E}\left[Z_{n}^{(3)}\right]\right)= & \lim _{n \rightarrow \infty} c[n, 3]\left(X_{n}^{(3)}-\mathbb{E}\left[X_{n}^{(3)}\right]\right) \\
& -3 \beta \lim _{n \rightarrow \infty} \frac{c[n, 3]}{c[n, 2]} c[n, 2]\left(Z_{n}-\mathbb{E}\left[Z_{n}\right]\right) \\
= & \tilde{Z} \quad \text { a.s. }
\end{aligned}
$$

Then assertion (iii) holds. 
For $\beta \geq 2$, define

$$
A_{n}^{2}:=\frac{1}{c[n, 2]^{2} \operatorname{var}\left[X_{n}\right]} \sim \begin{cases}\sigma_{\beta}^{-2} n^{(2-\beta) /(2+\beta)}, & \beta>2, \\ \frac{1}{72 \log n}, & \beta=2,\end{cases}
$$

where

$$
\sigma_{\beta}^{2}=\frac{8(1+\beta)^{2}(2+\beta)^{2}}{(\beta-2)(\beta-1) \beta^{2}}
$$

Lemma 2. If $\beta \geq 2$ then

$$
A_{n}^{2} \sum_{k=1}^{n} \frac{c[k, 2]^{2}}{S_{k-1}}\left(X_{k-1}^{(3)}-\mathbb{E}\left[X_{k-1}^{(3)}\right]\right) \stackrel{\mathbb{P}}{\rightarrow} 0 .
$$

Proof. Note that $c[n, 3]<c[n, 2]$. By assertions (i) and (ii) of Lemma 1 and Doob's inequality, we have

$$
\begin{aligned}
\mathbb{E}\left[\max _{1 \leq k \leq n}\left\{c[k-1,3]\left(X_{k-1}^{(3)}-\mathbb{E}\left[X_{k-1}^{(3)}\right]\right)\right\}^{2}\right] \\
\leq 2 \mathbb{E}\left[\max _{1 \leq k \leq n}\left\{c[k-1,3] T_{k-1}\right\}^{2}\right] \\
\quad+18 \mathbb{E}\left[\max _{1 \leq k \leq n}\left\{c[k-1,2]\left(X_{k-1}-\mathbb{E}\left[X_{k-1}\right]\right)\right\}^{2}\right] \\
\leq 8 c[n, 3]^{2} \operatorname{var}\left[X_{n}^{(3)}+3 X_{n}\right]+72 c[n, 2]^{2} \operatorname{var}\left[X_{n}\right]
\end{aligned}
$$

Hence, for $\beta \geq 2$,

$$
\begin{aligned}
\mathbb{P}\left(A_{n}^{2} \mid\right. & \left.\sum_{k=1}^{n} c[k, 2]^{2} \frac{X_{k-1}^{(3)}-\mathbb{E}\left[X_{k-1}^{(3)}\right]}{S_{k-1}} \mid \geq \varepsilon\right) \\
& \leq \frac{1}{\varepsilon^{2}} \mathbb{E}\left(A_{n}^{2} \sum_{k=1}^{n} c[k, 2]^{2} \frac{X_{k-1}^{(3)}-\mathbb{E}\left[X_{k-1}^{(3)}\right]}{S_{k-1}}\right)^{2} \\
& \leq \frac{1}{\varepsilon^{2}}\left(A_{n}^{2} \sum_{k=1}^{n} \frac{c[k, 2]^{2}}{c[k-1,3] S_{k-1}}\right)^{2} \mathbb{E}\left[\max _{1 \leq k \leq n}\left\{c[k-1,3]\left(X_{k-1}^{(3)}-\mathbb{E}\left[X_{k-1}^{(3)}\right]\right)\right\}^{2}\right] \\
& \leq O(1) A_{n}^{4}\left(c[n, 3]^{2} \operatorname{var}\left[X_{n}^{(3)}+3 X_{n}\right]+c[n, 2]^{2} \operatorname{var}\left[X_{n}\right]\right) \\
& \rightarrow 0
\end{aligned}
$$

which completes the proof.

For $-1<\beta<2$, define

$$
B_{n}^{2}:= \begin{cases}\frac{(2-\beta)(\beta-1) \beta^{2}}{8(1+\beta)^{2}(2+\beta)^{2}} n^{(2-\beta) /(2+\beta)}, & 1<\beta<2, \\ \frac{n^{1 / 3}}{96 \log n}, & \beta=1, \\ \frac{1}{4} n^{1 /(2+\beta)}, & -1<\beta<1 .\end{cases}
$$


Lemma 3. If $-1<\beta<2$,

$$
B_{n}^{2} \sum_{k=n+1}^{\infty} \frac{4 c[k, 2]^{2}}{S_{k-1}}\left(X_{k-1}^{(3)}-\mathbb{E}\left[X_{k-1}^{(3)}\right]\right) \stackrel{\mathbb{P}}{\rightarrow} \begin{cases}0, & 1 \leq \beta<2, \\ \tilde{Z}, & -1<\beta<1,\end{cases}
$$

where

$$
\tilde{Z}=\lim _{n \rightarrow \infty} c[n, 3]\left(Z_{n}^{(3)}-\mathbb{E}\left[Z_{n}^{(3)}\right]\right) \quad \text { a.s. }
$$

Proof. Since

$$
X_{k}^{(3)}-\mathbb{E}\left[X_{k}^{(3)}\right]=T_{k}-3\left(X_{k}-\mathbb{E}\left[X_{k}\right]\right),
$$

where $T_{k}$ is defined in (11), it is sufficient to show that

$$
B_{n}^{2} \sum_{k=n+1}^{\infty} \frac{c[k, 2]^{2}}{S_{k-1}}\left(X_{k-1}-\mathbb{E}\left[X_{k-1}\right]\right) \stackrel{\mathbb{P}}{\rightarrow} 0
$$

and

$$
B_{n}^{2} \sum_{k=n+1}^{\infty} \frac{4 c[k, 2]^{2}}{S_{k-1}} T_{k-1} \stackrel{\mathbb{P}}{\rightarrow} \begin{cases}0, & 1 \leq \beta<2, \\ \tilde{Z}, & -1<\beta<1 .\end{cases}
$$

It is easy to check that, for any $-1<\beta<2$,

$$
B_{n}^{2} \sum_{k=n+1}^{\infty} \frac{c[k, 2]^{2}}{S_{k-1} c[k-1,2]} \rightarrow 0 .
$$

Then, by assertion (i) of Lemma 1,

$$
\begin{aligned}
& \left|B_{n}^{2} \sum_{k=n+1}^{\infty} \frac{c[k, 2]^{2}}{S_{k-1}}\left(X_{k-1}-\mathbb{E}\left[X_{k-1}\right]\right)\right| \\
& \quad \leq\left(B_{n}^{2} \sum_{k=n+1}^{\infty} \frac{c[k, 2]^{2}}{S_{k-1} c[k-1,2]}\right)\left(\sup _{k \geq n}\left|c[k, 2]\left(X_{k}-\mathbb{E}\left[X_{k}\right]\right)\right|\right) \\
& \quad \rightarrow 0 \quad \text { a.s., }
\end{aligned}
$$

which, of course, implies (16).

Let

$$
\tilde{M}_{k}:=c[k, 3] T_{k}-c[k-1,3] T_{k-1}, \quad k \geq 1 .
$$

Then

$$
\begin{aligned}
B_{n}^{2} \sum_{k=n+1}^{\infty} \frac{4 c[k, 2]^{2}}{S_{k-1}} T_{k-1}= & B_{n}^{2}\left(\sum_{k=n+1}^{\infty} \frac{4 c[k, 2]^{2}}{S_{k-1} c[k-1,3]}\right) c[n, 3] T_{n} \\
& +B_{n}^{2} \sum_{k=n+2}^{\infty} \frac{4 c[k, 2]^{2}}{S_{k-1} c[k-1,3]} \sum_{i=n+1}^{k-1} \tilde{M}_{i} \\
= & : I_{1}(n)+I_{2}(n) .
\end{aligned}
$$

Since

$$
B_{n}^{2} \sum_{k=n+1}^{\infty} \frac{4 c[k, 2]^{2}}{S_{k-1} c[k-1,3]} \sim 4 B_{n}^{2} n^{-1 /(2+\beta)} \rightarrow \begin{cases}0, & 1 \leq \beta<2 \\ 1, & \beta<1,\end{cases}
$$


by the proof of assertion (iii) of Lemma 1, we have

$$
I_{1}(n) \stackrel{\mathbb{P}}{\rightarrow} \begin{cases}0, & 1 \leq \beta<2, \\ \tilde{Z}, & -1<\beta<1 .\end{cases}
$$

By (12), as $k \rightarrow \infty$, we have, for $-1<\beta<2$,

$$
\begin{aligned}
\mathbb{E}\left[\tilde{M}_{k}^{2}\right] & =c[k, 3]^{2} \operatorname{var}\left[T_{k}\right]-c[k-1,3]^{2} \operatorname{var}\left[T_{k-1}\right] \\
& =\left(\frac{c[k, 3]^{2} c[k-1,6]}{c[k-1,3]^{2} c[k, 6]}-1\right) c[k-1,3]^{2} \operatorname{var}\left[T_{k-1}\right]+c[k, 3]^{2} \tilde{b}_{k} \\
& =-\frac{9 c[k-1,3]^{2}}{\left(S_{k-1}+3\right)^{2}} \operatorname{var}\left[T_{k-1}\right]+c[k, 3]^{2} \tilde{b}_{k} \\
& =O\left(k^{-(3+\beta) /(2+\beta)}\right),
\end{aligned}
$$

where $\tilde{b}_{k}$ is defined in (13). Hence, as $n \rightarrow \infty$,

$$
\begin{aligned}
\mathbb{E}\left[I_{2}(n)\right]^{2} & =B_{n}^{4} \mathbb{E}\left(\sum_{k=n+2}^{\infty} \frac{4 c[k, 2]^{2}}{S_{k-1} c[k-1,3]} \sum_{i=n+1}^{k-1} \tilde{M}_{i}\right)^{2} \\
& =B_{n}^{4} \mathbb{E}\left(\sum_{i=n+1}^{\infty} \tilde{M}_{i} \sum_{k=i+1}^{\infty} \frac{4 c[k, 2]^{2}}{S_{k-1} c[k-1,3]}\right)^{2} \\
& =B_{n}^{4} \sum_{i=n+1}^{\infty} \mathbb{E}\left[\tilde{M}_{i}^{2}\right]\left(\sum_{k=i+1}^{\infty} \frac{4 c[k, 2]^{2}}{S_{k-1} c[k-1,3]}\right)^{2} \\
& =O(1) B_{n}^{4} n^{-3 /(2+\beta)} \\
& \rightarrow 0
\end{aligned}
$$

which implies that $I_{2}(n) \stackrel{\mathbb{P}}{\rightarrow} 0$. Then (17) holds. The proof of Lemma 3 is complete.

Proof of Theorem 1. The $-1<\beta<2$ case has been proved in Lemma 1. Here we consider only the cases $\beta=2$ and $\beta>2$. By (2) and (6), we only need to prove that

$$
\frac{X_{n}-\mathbb{E}\left[X_{n}\right]}{\sqrt{\operatorname{var}\left[X_{n}\right]}} \stackrel{\mathrm{D}}{\rightarrow} N(0,1) \text {. }
$$

Let

$$
M_{k}:=c[k, 2]\left(X_{k}-\mathbb{E}\left[X_{k}\right]\right)-c[k-1,2]\left(X_{k-1}-\mathbb{E}\left[X_{k-1}\right]\right), \quad k=1,2, \ldots,
$$

with $M_{0}=0$. Clearly, the process $\left\{M_{k}, k \geq 1\right\}$ is a martingale difference sequence. By Corollary 3.1 of Hall and Heyde (1980), it is sufficient to show that, for any $\varepsilon>0$,

$$
\sum_{k=0}^{n} \mathbb{E}\left[\left(A_{n} M_{k}\right)^{2} I\left(\left|A_{n} M_{k}\right|>\varepsilon\right) \mid \mathcal{F}_{k-1}\right] \stackrel{\mathbb{P}}{\rightarrow} 0
$$

and

$$
\sum_{k=0}^{n} \mathbb{E}\left[\left(A_{n} M_{k}\right)^{2} \mid \mathcal{F}_{k-1}\right] \stackrel{\mathbb{P}}{\rightarrow} 1,
$$

where $A_{n}^{2}$ is defined in (14). 
For (19), we only need to prove that

$$
A_{n} \max _{1 \leq k \leq n}\left|M_{k}\right| \stackrel{\mathbb{P}}{\rightarrow} 0
$$

Note that $|c[n, 2]-c[n-1,2]| \leq c[n, 2] / n$ and $c[n, 2]\left(\mathbb{E}\left[X_{n}\right]-\mathbb{E}\left[X_{n-1}\right]\right) \rightarrow 0$. By (3), we have

$$
\begin{aligned}
\max _{1 \leq k \leq n}\left|M_{k}\right|= & \max _{1 \leq k \leq n} \mid(c[k, 2]-c[k-1,2])\left(X_{k-1}-\mathbb{E}\left[X_{k-1}\right]\right) \\
& +c[k, 2]\left(2 W_{k-1, V_{k}}+2+2 \beta+\beta^{2}\right)-c[k, 2]\left(\mathbb{E}\left[X_{k}\right]-\mathbb{E}\left[X_{k-1}\right]\right) \mid \\
\leq & \max _{0 \leq k \leq n-1}\left|\frac{c[k, 2]}{k}\left(X_{k}-\mathbb{E}\left[X_{k}\right]\right)\right|+2 \max _{0 \leq j<k \leq n-1}\left\{c[k, 2] W_{k-1, j}\right\}+O(1) .
\end{aligned}
$$

Thus, to prove (19), we only need to prove that

$$
A_{n} \max _{0 \leq k \leq n-1}\left|\frac{c[k, 2]}{k}\left(X_{k}-\mathbb{E}\left[X_{k}\right]\right)\right| \stackrel{\mathbb{P}}{\rightarrow} 0
$$

and

$$
A_{n} \max _{0 \leq j<k \leq n-1}\left\{c[k, 2] W_{k-1, j}\right\} \stackrel{\mathbb{P}}{\rightarrow} 0
$$

Since, for large $n$,

$$
\begin{aligned}
\max _{1 \leq k \leq n-1}\left|\frac{c[k, 2]}{k}\left(X_{k}-\mathbb{E}\left[X_{k}\right]\right)\right| \leq & \max _{1 \leq k \leq \log n}\left\{c[k, 2]\left|X_{k}-\mathbb{E}\left[X_{k}\right]\right|\right\} \\
& +\frac{1}{\log n} \max _{1 \leq k \leq n-1}\left\{c[k, 2]\left|X_{k}-\mathbb{E}\left[X_{k}\right]\right|\right\}
\end{aligned}
$$

it follows by Kolmogorov's inequality for the martingale sequence and (10) that, for any $\varepsilon>0$,

$$
\begin{aligned}
& \mathbb{P}\left(\max _{0 \leq k \leq n-1}\left|\frac{c[k, 2]}{k}\left(X_{k}-\mathbb{E}\left[X_{k}\right]\right)\right| \geq \varepsilon A_{n}^{-1}\right) \\
& \leq \mathbb{P}\left(\max _{1 \leq i \leq\lfloor\log n\rfloor}\left\{c[i, 2]\left|X_{i}-\mathbb{E}\left[X_{i}\right]\right|\right\} \geq \frac{\varepsilon}{2} A_{n}^{-1}\right) \\
&+\mathbb{P}\left(\max _{1 \leq i \leq n-1}\left\{c[i, 2]\left|X_{i}-\mathbb{E}\left[X_{i}\right]\right|\right\} \geq \frac{\varepsilon}{2} A_{n}^{-1} \log n\right) \\
& \leq \frac{4 c[\lfloor\log n\rfloor, 2]^{2} \operatorname{var}\left[X_{\lfloor\log n\rfloor}\right]}{\varepsilon^{2} A_{n}^{-2}}+\frac{4 c[n, 2]^{2} \operatorname{var}\left[X_{n}\right]}{\varepsilon^{2} A_{n}^{-2} \log ^{2} n} \\
& \rightarrow 0
\end{aligned}
$$

as $n \rightarrow \infty$. Then (22) holds.

Write

$$
M[n, n]:=c[n, 1] \max _{0 \leq j \leq n} W_{n, j} \geq c[n, 2] \max _{0 \leq j \leq n} W_{n, j} .
$$

For any $\beta>-1$, by the proof of Theorem 3.1 of Móri (2005), the process $\{M[n, n], n \geq 1\}$ is a nonnegative submartingale and, for any $m>2+\beta$,

$$
\sup _{n} \mathbb{E}\left[M[n, n]^{m}\right]<\infty .
$$


Then, for any $\varepsilon>0$, by Kolmogorov's inequality,

$$
\begin{aligned}
\mathbb{P}\left(A_{n} \max _{0 \leq j \leq k \leq n}\left\{c[k, 2] W_{k, j}\right\}>\varepsilon\right) & \leq \mathbb{P}\left(A_{n} \max _{0 \leq k \leq n} M[k, k]>\varepsilon\right) \\
& \leq \varepsilon^{-m} A_{n}^{m} \mathbb{E}(M[n, n])^{m} \\
& \rightarrow 0,
\end{aligned}
$$

which, together with the fact that $c[n, 2]<c[n-1,2]$ for any $n \geq 1$, implies (23). Hence, the conditional Lindeberg condition (19) has been verified.

By (3),

$$
\begin{aligned}
\mathbb{E}\left[\left(X_{n}-X_{n-1}-\left(2+2 \beta+\beta^{2}\right)\right)^{2} \mid \mathcal{F}_{n-1}\right] & =4 \mathbb{E}\left[W_{V_{n}, n-1}^{2} \mid \mathcal{F}_{n-1}\right] \\
& =4 \sum_{k=1}^{n-1} \frac{W_{n-1, k}^{3}}{S_{n-1}} \\
& =\frac{4}{S_{n-1}} X_{n-1}^{(3)} .
\end{aligned}
$$

Note that

$$
\begin{aligned}
\mathbb{E}[( & \left.\left.X_{n}-X_{n-1}-\left(2+2 \beta+\beta^{2}\right)\right)^{2} \mid \mathcal{F}_{n-1}\right] \\
= & \mathbb{E}\left[\left(X_{n}-\mathbb{E}\left[X_{n}\right]-X_{n-1}+\mathbb{E}\left[X_{n-1}\right]\right)^{2} \mid \mathcal{F}_{n-1}\right] \\
& +\left(\mathbb{E}\left[X_{n}\right]-\mathbb{E}\left[X_{n-1}\right]-\left(2+2 \beta+\beta^{2}\right)\right)^{2} \\
& +2\left(\mathbb{E}\left[X_{n}\right]-\mathbb{E}\left[X_{n-1}\right]-\left(2+2 \beta+\beta^{2}\right)\right) \mathbb{E}\left[X_{n}-\mathbb{E}\left[X_{n}\right]-X_{n-1}+\mathbb{E}\left[X_{n-1}\right] \mid \mathcal{F}_{n-1}\right] \\
= & \mathbb{E}\left[\left(X_{n}-\mathbb{E}\left[X_{n}\right]\right)^{2} \mid \mathcal{F}_{n-1}\right]+\left(1-\frac{2 c[n-1,2]}{c[n, 2]}\right)\left(X_{n-1}-\mathbb{E}\left[X_{n-1}\right]\right)^{2} \\
& +4\left(\frac{\mathbb{E}\left[X_{n-1}\right]}{S_{n-1}}\right)^{2}+8 \frac{\mathbb{E}\left[X_{n-1}\right]}{S_{n-1}^{2}}\left(X_{n-1}-\mathbb{E}\left[X_{n-1}\right]\right) .
\end{aligned}
$$

Then

$$
\begin{aligned}
\sum_{k=1}^{n} \mathbb{E}[ & \left.M_{k}^{2} \mid \mathcal{F}_{k-1}\right] \\
= & \sum_{k=1}^{n}\left(c[k, 2]^{2} \mathbb{E}\left[\left(X_{k}-\mathbb{E}\left[X_{k}\right]\right)^{2} \mid \mathcal{F}_{k-1}\right]-c[k-1,2]^{2}\left(X_{k-1}-\mathbb{E}\left[X_{k-1}\right]\right)^{2}\right) \\
= & \sum_{k=1}^{n} \frac{4 c[k, 2]^{2}}{S_{k-1}} X_{k-1}^{(3)}-\sum_{k=1}^{n} \frac{4 c[k, 2]^{2}}{S_{k-1}^{2}}\left(X_{k-1}-\mathbb{E}\left[X_{k-1}\right]\right)^{2} \\
& -4 \sum_{k=1}^{n}\left(\frac{c[k, 2] \mathbb{E}\left[X_{k-1}\right]}{S_{k-1}}\right)^{2}-8 \sum_{k=1}^{n} \frac{c[k, 2]^{2} \mathbb{E}\left[X_{k-1}\right]}{S_{k-1}^{2}}\left(X_{k-1}-\mathbb{E}\left[X_{k-1}\right]\right) \\
= & \sum_{k=1}^{n} \frac{4 c[k, 2]^{2}}{S_{k-1}}\left(X_{k-1}^{(3)}-\mathbb{E}\left[X_{k-1}^{(3)}\right]\right)-\sum_{k=1}^{n} \frac{4 c[k, 2]^{2}}{S_{k-1}^{2}}\left(X_{k-1}-\mathbb{E}\left[X_{k-1}\right]\right)^{2} \\
& -8 \sum_{k=1}^{n} \frac{c[k, 2]^{2} \mathbb{E}\left[X_{k-1}\right]}{S_{k-1}^{2}}\left(X_{k-1}-\mathbb{E}\left[X_{k-1}\right]\right)+\sum_{k=1}^{n} c[k, 2]^{2} \hat{b}_{k} \\
= & : I I_{1}(n)+I I_{2}(n)+I I_{3}(n)+I I_{4}(n) .
\end{aligned}
$$


It follows by Lemma 2 that $A_{n}^{2} I I_{1}(n) \stackrel{\mathbb{P}}{\rightarrow} 0$. By Chebyshev's inequality, for any $\varepsilon>0$,

$$
\mathbb{P}\left(A_{n}^{2} \sum_{k=1}^{n} \frac{c[k, 2]^{2}}{S_{k-1}^{2}}\left(X_{k-1}-\mathbb{E}\left[X_{k-1}\right]\right)^{2}>\varepsilon\right) \leq \frac{A_{n}^{2}}{\varepsilon} \sum_{k=1}^{n} \frac{c[k, 2]^{2}}{S_{k-1}^{2}} \operatorname{var}\left[X_{k-1}\right] \rightarrow 0,
$$

which implies that $A_{n}^{2} I I_{2}(n) \stackrel{\mathbb{P}}{\rightarrow} 0$. Note that, for any $\beta \geq 2$, the limit

$$
\lim _{n \rightarrow \infty} A_{n}^{2} \sum_{k=1}^{n} \frac{c[k, 2]^{2}\left(\mathbb{E}\left[X_{k-1}\right]\right)^{2}}{S_{k-1}^{2}}=: L_{\beta}
$$

exists and is positive, and, for any $\varepsilon>0$,

$$
\frac{\left|I I_{3}(n)\right|}{8} \leq \frac{1}{2}\left(\frac{4 L_{\beta}}{\varepsilon} \frac{I I_{2}(n)}{4}+\frac{\varepsilon}{4 L_{\beta}} \sum_{k=1}^{n} \frac{c[k, 2]^{2}\left(\mathbb{E}\left[X_{k-1}\right]\right)^{2}}{S_{k-1}^{2}}\right) .
$$

Then, for any $\varepsilon>0$ and sufficiently large $n$,

$$
\mathbb{P}\left(A_{n}^{2}\left|I I_{3}(n)\right|>8 \varepsilon\right) \leq \mathbb{P}\left(\frac{L_{\beta}}{\varepsilon} A_{n}^{2} I I_{2}(n)+\varepsilon>2 \varepsilon\right) \leq \mathbb{P}\left(A_{n}^{2} I I_{2}(n)>\frac{\varepsilon^{2}}{L_{\beta}}\right) \rightarrow 0,
$$

which also implies that $A_{n}^{2} I I_{3}(n) \stackrel{\mathbb{P}}{\rightarrow} 0$. Finally, it is easy to check that $\lim _{n \rightarrow \infty} A_{n}^{2} I I_{4}(n)=1$ for all $\beta \geq 2$. Hence, (20) holds. This completes the proof of Theorem 1.

Proof of Theorem 2. Note that, for $-1<\beta<2$,

$$
Z-c[n, 2]\left(Z_{n}-\mathbb{E}\left[Z_{n}\right]\right)=\sum_{k=n+1}^{\infty} M_{k}
$$

where $M_{k}$ is defined in (18). By Corollary 3.1 of Hall and Heyde (1980), in order to prove Theorem 2, it is sufficient to show that, for any $\varepsilon>0$,

$$
\sum_{k=n+1}^{\infty} \mathbb{E}\left[\left(B_{n} M_{k}\right)^{2} I\left(\left|B_{n} M_{k}\right|>\varepsilon\right) \mid \mathcal{F}_{k-1}\right] \stackrel{\mathbb{P}}{\rightarrow} 0
$$

and

$$
\sum_{k=n+1}^{\infty} \mathbb{E}\left[\left(B_{n} M_{k}\right)^{2} \mid \mathcal{F}_{k-1}\right] \stackrel{\mathbb{P}}{\rightarrow} \begin{cases}1, & 1 \leq \beta<2, \\ \tilde{Z}+C_{2}, & \beta<1,\end{cases}
$$

where $B_{n}$ and $C_{2}$ are defined in (15) and (8), respectively.

To prove (25), we only need to prove that

$$
B_{n} \max _{k \geq n+1}\left|M_{k}\right| \stackrel{\mathbb{P}}{\rightarrow} 0 .
$$

For any $\beta>-1$, by Theorem 3.1 of Móri (2005),

$$
\lim _{n \rightarrow \infty}\left(n^{-1 /(2+\beta)} \max _{0 \leq j \leq n} W_{n, j}\right)
$$


exists a.s. and the limit is positive and finite a.s. By the proof of Theorem 1 , we have $c[n, 2]\left(X_{n}-\right.$ $\left.\mathbb{E}\left[X_{n}\right]\right) \rightarrow Z$ a.s. for any $-1<\beta<2$. Hence, similarly to (21), for $-1<\beta<2$, we have

$$
\begin{aligned}
\max _{k \geq n+1}\left|M_{k}\right| \leq & \max _{k \geq n}\left|\frac{c[k, 2]}{k}\left(X_{k}-\mathbb{E}\left[X_{k}\right]\right)\right|+2 \max _{k \geq n+1}\left\{c[k, 2] \max _{0 \leq j \leq k-1} W_{k-1, j}\right\} \\
& +\max _{k \geq n+1}\left\{c[k, 2]\left|\left(2+2 \beta+\beta^{2}\right)-\left(\mathbb{E}\left[X_{k}\right]-\mathbb{E}\left[X_{k-1}\right]\right)\right|\right\} \\
\leq & O\left(\frac{1}{n}\right)+O\left(n^{-1 /(2+\beta)}\right)+O(1) n^{-2 /(2+\beta)} \quad \text { a.s. }
\end{aligned}
$$

Now (27) follows directly and (25) holds.

Next we will prove (26). Similarly to (24), we have

$$
\begin{aligned}
\sum_{k=n+1}^{\infty} \mathbb{E}\left[M_{k}^{2} \mid \mathcal{F}_{k-1}\right]= & \sum_{k=n+1}^{\infty} \frac{4 c[k, 2]^{2}}{S_{k-1}}\left(X_{k-1}^{(3)}-\mathbb{E}\left[X_{k-1}^{(3)}\right]\right) \\
& -\sum_{k=n+1}^{\infty} \frac{4 c[k, 2]^{2}}{S_{k-1}^{2}}\left(X_{k-1}-\mathbb{E}\left[X_{k-1}\right]\right)^{2}+\sum_{k=n+1}^{\infty} c[k, 2]^{2} \hat{b}_{k} \\
= & : I I I_{1}(n)+I I I_{2}(n)+I I I_{3}(n) .
\end{aligned}
$$

It is also easy to check that

$$
B_{n}^{2} I I I_{3}(n) \rightarrow \begin{cases}1, & 1 \leq \beta<2 \\ C_{2}, & -1<\beta<1\end{cases}
$$

By Chebyshev's inequality, for any $\varepsilon>0$,

$$
\mathbb{P}\left(B_{n}^{2} \sum_{k=n+1}^{\infty} \frac{4 c[k, 2]^{2}}{S_{k-1}^{2}}\left(X_{k-1}-\mathbb{E}\left[X_{k-1}\right]\right)^{2}>\varepsilon\right) \leq \frac{B_{n}^{2}}{\varepsilon} \sum_{k=n+1}^{\infty} \frac{4 c[k, 2]^{2}}{S_{k-1}^{2}} \operatorname{var}\left[X_{k-1}\right] \rightarrow 0,
$$

which implies that $B_{n}^{2} I I I_{2}(n) \stackrel{\mathbb{P}}{\rightarrow} 0$. Now (26) follows by Lemma 3. This completes the proof of Theorem 2.

\section{The variants}

Plane-oriented recursive trees are nearly identical to the Barabási-Albert random trees. In the case the tree is embedded in the plane, the left-to-right order of the children of the nodes is relevant (see, for example, Mahmoud et al. (1993)). The outdegree instead of the degree is considered here. It is equal to the degree of the node minus one, except for the root, where it is the same as the degree. If a vertex $v$ has outdegree $d$ then there are $d+1$ possible ways to attach a new vertex to $v$. We get the plane-oriented recursive trees model if all these possibilities are equally likely. Namely, the probability that a vertex of outdegree $d$ gets the new edge is proportional to $d+1$. In the generalized plane-oriented recursive trees model, this probability is proportional to $d+\beta$ for some $\beta>0$ (see, for example, Drmota (2009, p. 255)). One can get the analogous results by our method. We omit the details.

A path in a graph is a sequence of adjacent edges, which do not pass through the same vertex more than once, and the length of the path is the number of edges in it. The degree of an edge is equal to the number of its adjacent edges. For a simple graph $G$, the Gordon-Scantlebury index of $G$ is equal to the number of paths of length two in $G$ (see Gordon and Scantlebury (1964)), and the Platt index is equal to the total sum of edge degrees in $G$ (see Platt (1947)). 
The Zagreb index is related to the Gordon-Scantlebury and Platt indices (see, for example, Barysz et al. (1986)). Let $Z_{G}, S_{G}$, and $P_{G}$ be the Zagreb index, the Gordon-Scantlebury index, and the Platt index of graph $G$, respectively. Nikolić et al. (2003) showed that

$$
Z_{G}=2\left(S_{G}+E(G)\right) \quad \text { and } \quad P_{G}=2 S_{G}
$$

where $E(G)$ denotes the number of edges in $G$. Since the number of edges in a tree of size $n$ is obviously $n-1$, one can directly establish parallel results for the Gordon-Scantlebury and Platt indices of a scale-free tree. We also omit these details.

\section{Acknowledgements}

The work of the first author was supported by the NSFC (grant number 11101395) and the Specialized Research Fund for the Doctoral Program of Higher Education of China (grant number 20093402120014). The work of the second author was supported by the NSFC (grant number 10801122) and the RFDP (grant number 200803581009).

\section{References}

Albert, R. And Barabási, A.-L. (2002). Statistical mechanics of complex networks. Rev. Modern Phys. 47, 47-97. Andova, V. et al. (2011). On the Zagreb index inequality of graphs with prescribed vertex degrees. Discrete Appl. Math. 159, 852-858.

Barabási, A. -L. AND Albert, R. (1999). Emergence of scaling in random networks. Science 286, 509-512.

Barysz, M., Plavšić, D. And Trinajstić, N. (1986). A note on topological indices. MATCH Commun. Math. Comput. Chem. 19, 89-116.

Bollobás, B. AND Riordan, O. (2004). The diameter of a scale-free random graph. Combinatorica 24, 5-34.

Bollobás, B., Riordan, O., Spencer, J. And Tusnády, G. (2001). The degree sequence of a scale-free random graph process. Random Structures Algorithms 18, 279-290.

Dondajewski, M. AND Szymański, J. (2009). Branches in scale-free trees. J. Math. Sci. 161, 961-968.

Dorogovtsev, S. N. And Mendes, J. F. F. (2003). Evolution of Networks. Oxford University Press.

Drmota, M. (2009). Random Trees. Springer Wien New York, Vienna.

Durrett, R. (2007). Random Graphs Dynamics. Cambrige University Press.

Feng, Q. And Hu, Z. (2011). On the Zagreb index of random recursive trees. J. Appl. Prob. 48, 1189-1196.

Gordon, M. And ScAntlebury, G. R. (1964). Non-random polycondensation: statistical theory of the substitution effect. Trans. Faraday Soc. 60, 604-621.

Hall, P. And Heyde, C. C. (1980). Martingale Limit Theory and Its Application. Academic Press, New York.

JANSON, S. (2005). Asymptotic degree distribution in random recursive trees. Random Structures Algorithms $26,69-83$.

Katona, Z. (2005). Width of a scale-free tree. J. Appl. Prob. 42, 839-850.

Katona, Z. (2006). Levels of a scale-free tree. Random Structures Algorithms 29, 194-207.

Kim, D.-H., Noh, J. D. And Jeong, H. (2004). Scale-free trees: the skeletons of complex networks. Phys. Rev. E 70, 046126, 5 pp.

LI, X., LI, Z. AND WANG, L. (2003). The inverse problems for some topological indices in combinatorial chemistry. J. Comput. Biol. 10, 47-55.

Mahmoud, H., Smythe, R. and Szymański, J. (1993). On the structure of plane-oriented recursive trees and their branches. Random Structures Algorithms 4, 151-176.

Móri, T. F. (2002). On random trees. Studia Sci. Math. Hung. 39, 143-155.

Móri, T. F. (2005). The maximum degree of the Barabási-Albert random trees. Combinatorics Prob. Comput. 14, 339-348.

Neininger, R. (2002). The Wiener index of random trees. Combinatorics Prob. Comput. 11, 587-597.

Nikiforov, V. (2007). The sum of the squares of degrees: sharp asymptotics. Discrete Math. 307, 3187-3193.

Nikolić, S., KovačEvić, G., Miličević, A. And Trinajstić, N. (2003). The Zagreb indices 30 years after. Croatica Chemica ACTA 76, 113-124.

Peled, U. N., Petreschi, R. And Sterbini, A. (1999). ( $n, e)$-graphs with maximum sum of squares of degrees. J. Graph Theory 31, 283-295.

Platt, J. R. (1947). Influence of neighbor bonds on additive bond properties in paraffins. J. Chem. Phys. 15, 419-420. 